\title{
Mobile Phone Mood Charting for Adolescents ${ }^{1}$
}

\author{
Mark Matthews, Dr. Gavin Doherty \\ Trinity College Dublin \\ Dr. John Sharry, Prof. Carol Fitzpatrick \\ Mater Hospital, Dublin
}

\begin{abstract}
Mobile phones may provide a useful and engaging platform for supporting therapeutic services working with adolescents. This paper examines the potential benefits of the mobile phone for self-charting moods in comparison to existing methods in current practice. The paper describes a mobile phone application designed by the authors which allows adolescents to record moods on their personal mobile phones. The authors propose that a personal mobile device is more suitable for adolescents than other devices. A pilot study with a non-clinical adolescent group in schools comparing a mobile and a paper diary is presented, along with discussion and analysis of the results. The main finding of the study was that compliance is significantly higher on mobile phones than paper, and the task was not found to be any more difficult to complete using mobile phones than with paper charts. A number of salient issues requiring further research are also identified.
\end{abstract}

\section{Introduction}

Mental health professionals often experience difficulties engaging adolescent clients in therapeutic activities (Offer, 1991, pp. 623-630; US Surgeon General, 1999). This is especially the case between therapeutic sessions when clients are asked to complete 'homework' activities which require them to prepare materials in advance of sessions or to chart their feelings between them. Typically there is a very low rate of task completion associated with these activities. This might be attributed to the use of materials that do not sufficiently engage an adolescent audience or take into account their specific needs as a group.

The mobile phone is a device which adolescents frequently use and are familiar with. As a platform clients may find it more engaging and it potentially enables increased accessibility to therapeutic activities and materials. There has been very little research exploring the mobile phone as a therapeutic platform for adolescents. This paper proposes and examines the mobile phone as a platform for engaging adolescents in therapeutic activities. It will look particularly at mood charting as an example to explore issues related to employing the mobile phone in this way. It begins with an analysis of current methods. A new mobile system for adolescent mood charting, developed by the authors, is presented along with the results of an initial pilot study. This research has also allowed an initial exploration of the general benefits and limitations of using the mobile phone for therapeutic activities with an adolescent audience. Throughout the authors make the distinction between mobile devices, essentially portable computers and

\footnotetext{
${ }^{1}$ Preprint of paper published as: M. Matthews, G. Doherty and J. Sharry, Mobile Phone Mood Charting for Adolescents, British Journal of Guidance and Conselling, 36(2), pp.113 - 129, 2008. DOI 10.1080/03069880801926400.
} 
personal mobile devices, portable computers which the owner has a close relationship with, knows how to use and strongly values. This paper argues that personal mobile devices, particularly the mobile phone, have many advantages over other mobile devices for use by therapeutic services working with adolescents.

Mood Charting

Mood charting - also known as "symptom charting" or "mood monitoring" - is used in therapeutic treatments for a wide range of complaints, including bipolar disorder, attention deficit hyperactive disorder (ADHD), obsessive compulsive disorder (OCD) and non-verbal learning disorder (NVLD). It is an important component of Cognitive Behavioural Therapy (CBT). Several studies have found that regular and reliable client self-charting has a positive effect on therapeutic outcomes (M. Bauer et al., 2005, 1-7; Leverich \& Post, 1996, 48-61). Mood charts involve clients recording their moods at regular intervals to help identify contributing factors to their emotional state and behaviours. They provide a method to track clients' symptoms, behaviours and feelings on a regular basis. Mood charts provide an account of the emotional state of clients during their daily lives outside of therapeutic sessions. This information can be used to identify triggers of moods and help develop coping strategies. They may help clients develop greater self-awareness, affording them subsequent control and understanding of their behaviour. By gathering therapeutic content between sessions they may save practitioners' time. They assist in identifying any affects a change in medication might have on a client and they help provide an emotional history of the client.

Mood charting often involves the client recording other factors such as energy levels, medication taken or the amount of hours slept. The make up of a mood chart can vary depending on the client. Practitioners often find it useful to have clients record other factors like anger outbursts, alcohol consumption or negative thoughts. Various scales have been used to record moods. For example, both ChronoRecord (M. Bauer et al., 2004, 67-74), a computer-based chart, and Life Chart Method (Post et al., 2001, 169-176) use a visual analogue scale between 1-100. Some charts use 7 point textual labels. Chronosheet, a paper chart uses a 100mm visual analogue scale between extreme states. Charts designed for parents to chart their children's moods typically provide more detailed and consistent information and are not required to be particularly visually appealing. Self-reporting charts for adolescents need to be more easily understood and engaging.

The following sections will briefly examine current methods for recording moods. These have been dealt with in more depth elsewhere (Bolger et al., 2003, 579-616). Koop provides a detailed review of the use of mobile computers in clinical trials (Koop, 2002, 9-24).

\section{Paper-based mood charting}

"Pen-and-paper" charting is the most widely used method for charting moods in current clinical practice. The main benefit of paper-based mood charting is its ease of use. Adolescents are familiar with pen-and-paper activities and they are straightforward for practitioners to implement. No technical training is required. It is an inclusive method available to the majority of clients with basic literacy skills. It also allows a degree of creativity and individuality in the expression of moods and feelings, by enabling the client to draw the feeling or thought instead of using words to describe them. Paper-based mood charting is low in resource costs. Charts can be easily reproduced using a photocopier or a computer printer.

Significant limitations to paper-based charting have been identified by several independent studies. Several studies have shown that paper-based charts have very low compliance rates 
(Feldman Barrett \& Barrett, 2001, 175-185; Stone et al., 2002, 1193-1194). Paper diaries are often completed retrospectively. Shiffman and colleagues have shown that recollection is a constructive process that does not always represent actual events (Shiffman et al., 1997, 292300). The major difficulty with paper-based methods is that it is usually impossible for a practitioner to know whether the data has been distorted by the client in these ways. Entering this data into a computer absorbs significant time and may result in errors. Paper charts may be difficult to fill out unobtrusively, and so it can be argued that this method does not offer clients sufficient privacy in a family, school or peer environment. For example, it may be possible for someone to look over the shoulder of a client filling in a mood diary on a paper chart. Furthermore, the information recorded is not satisfactorily secure, and extra measures and responsibility are required by the client to keep it safe, such as locking the chart in a drawer. If the client does not feel that their chart is safe from other people, then they may be unlikely to record their moods honestly. If the chart is lost then all the charted data is lost. Clients' access to charting is limited. Few adolescents carry pen and paper around with them. Should they remember to record a mood entry when they are out without pen and paper, then they have to wait until they have their mood chart to record an entry.

Although significant limitations to paper mood charts have been identified, they continue to provide a low-tech and relatively low-cost solution to mood charting which has yet to be made more convenient and straightforward by any other method.

\section{Electronic Diaries}

An electronic diary can be used to provide a computerized mood chart. Over the past ten years, electronic diaries have become available and been increasingly used in clinical studies see for example (Raymond \& Ross, 2000, 48-57; Tseng et al., 1998, 599-610). The two existing hardware technologies currently used are desktop computers and mobile computers. Electronic diaries have advantages over paper-based charting methods. They provide greater data quality than paper-based charting, because they can reliably record exactly when the client enters mood data. This is often referred to as "time-stamping". It improves the quality of the data collected, and in removing the need to make a judgement on the validity of the information, can save the practitioner's time (M. Bauer et al., 2005, 1-7). By setting an automatic reminder that encourages the client to chart, an electronic diary can alleviate some of the responsibility on the client to remember to chart (Bolger et al., 2003, 579-616). The effectiveness of reminders is dependent on how much contact the client has with the signalling device. Electronic devices that are connected to a network (such as the internet) can transmit charted information to a secure location, which is then accessible to practitioners during a session. They also remove the requirement for practitioners to manually enter charted information. An electronic diary can allow the practitioners to manipulate and transform the information according to chosen parameters. For example, with an electronic system the practitioner may be able to print daily/monthly/yearly graphical charts at the touch of a button. This gives practitioners a convenient overview of a client's situation without requiring extra time or expense.

Unlike paper-based mood charts, digital technologies can offer the client a higher degree of security. Access to the charts can be password-protected and data can be encrypted. Paradoxically, although paper charts provide little security, practitioners often express more concern about the security provided by electronic systems. This may be due, in part, to the lack of transparency in how they handle (client) information. It is possible decrypt encrypted data. The level of security required for any system is generally balanced against the value of the 
information to the person trying to break the security. This must be taken into account when designing computer systems, particularly for adolescents. There is generally no reason to, and software designers must be careful not to, record or transmit client-identifying information. There is no particular need for, or benefit from, clients recording identifying information like their name or address. Practitioners are interested in information related to mood. Existing paper charts do not contain identifying information. For adolescent clients their greatest concern is that family or peers will get access to their mood charts. Lost paper charts could be identified by peers or family members by a client's hand-writing, an identifying factor. A lost electronic device can be password-protected. Even if the password is broken, the information does not necessarily identify the client. When introducing an electronic diary, it is important to provide clear protocols and explanations about how information is dealt with and what security provisions are in place.

Personal Digital Assistants (PDAs) are small portable hand-held devices which several studies have used to administer electronic diaries. Research has shown that this method overcomes many of the limitations of paper-based charts. For example, Bauer and colleagues explored this issue for adult patients with bipolar disorder self-reporting mood on a desktop computer (M. Bauer et al., 2005, 1-7). More generally, VanDenKerkhof found that PDAs were an efficient and comfortable tool for patients to complete patient questionnaires by comparison with paper questionnaires (VanDenKerkhof et al., 2005, 1075-1080). Studies report greater subject compliance rates using PDAs in comparison to paper-based charts (Raymond \& Ross, 2000, 48-57).

Although PDAs have been effective in clinical studies there are drawbacks and limitations to using them in day-to-day clinical practice as underlined by their lack of widespread use. Training and technical support may be required for adolescents, most of whom would never have used a PDA before. In one study using PDAs, adolescent subjects were given training sessions and an instruction manual and there was a technician on-hand during the testing period in case anything went wrong with the device (Henker et al., 2002, 660-670). The PDA is not a commonly used by adolescents, and so it is difficult for the client to record their moods in an unobtrusive fashion. Finally, the cost of a PDA is still prohibitive in a clinical situation.

The Mobile Phone as a Therapeutic Platform

Research has shown that client-centred approaches to psychotherapy which engage the interests and strengths of clients are more likely to be successful (Assay \& Lambert, 1999, 2355). Technology such as computer games and mobile phones hold great appeal for adolescents. The mobile phone represents a promising client-centred platform, which may provide access to adolescents' personal space and offer them persistent and continual access to therapeutic materials and activities outside of the therapy session. The mobile phone is a robust networked device that is designed to transmit and receive data. It is distinct from other mobile devices, like PDAs, in that it is a uniquely personal device. Adolescent ownership of mobile phones throughout Europe is widespread. This has been extensively documented. For example, Norway is reported to have almost $100 \%$ saturation of mobile phones in both sexes between the ages of 13-20 (Ling, 2001). 90\% of young adults in the UK have been reported to have mobile phones (Crabtree et al., 2003). Similar figures are found for pre-teens and adolescents across Europe, see for example (van Veen, 2007).

Mobile Phone in Mental Health

Research into using the mobile phone as a therapeutic support device is at an initial stage. A 
study using SMS text messages in the aftercare of bulimia nervosa adult patients outlined the following benefits of mobile technology (S. Bauer et al., 2003, 279-290): (1) access to this technology is independent of space and time, (2) there is little temporal or monetary cost, (3) it is an interactive medium, (4) patients are encouraged to express themselves briefly and concisely, (5) the medium allows patients to stay in contact with therapy and (6) it can help to bridge the cap between inpatient and outpatient treatment.

Using the mobile phone may result in adolescents thinking more about therapeutic matters. One study, which used PDAs as an extension to therapy, reported that signals from the computer reminded clients to think about things like eating behaviour and mood (Norton et al., 2003, 231242). Research into mobile mood charting should build on the research into the mobile phone in related areas, such as education, and identify aspects of this platform that are pertinent to supporting to mental health interventions.

A successful implementation using mobile technology might support the therapeutic process by: (1) creating content for use in a session, (2) engaging adolescents in beneficial therapeutic activities, (3) encouraging them to spend more time thinking about therapeutic matters and (4) making therapeutic material more convenient and accessible.

\section{Mobile Mood Charting}

This paper uses the term 'Mobile Mood Charting' to refer to clients using their own mobile phones to chart their moods. We shall now assess the mobile phone as a platform for mood charting.

One important advantage that mobile diaries have over all other charting methods is that the mobile phone is already available to many adolescents at almost any time and any place. There is no need for clinics to purchase or maintain mobile devices. In a study of adolescent and young adults usage of mobile phones, Geser reports that almost all participants have their mobile phone turned on all day (Geser, 2006). In its 2004 report the ITU found that most adolescents keep their phone with them all day and that many are afraid to leave home without it; at night many keep it under their pillows or on bedside tables (International Telecommunications Union, 2004). The convenience of a mobile phone may reduce the client's responsibility to remember to chart. The mobile phone provides many opportunities when adolescents can record their moods. A mobile diary may have unique access to an adolescent's idle time; for example when waiting for something or bored. Time waiting at a bus stop or queuing in a shop may become an opportunity to chart.

The mobile phone shares many of the advantages of other electronic diaries. It is straightforward to appraise compliance by recording a timestamp when an entry is recorded. Data entry costs are removed and it is possible to remind clients using electronic signalling. Signalling on a personal mobile device may be less invasive than other methods. In order to remind clients to chart, an automated text message can be sent to them at an agreed time every day. Adolescents may be more likely to respond to a 'New message' signal from their mobile phone. Unlike other mobile devices where a signalling message may attract peer attention, a reminder message is indistinguishable from any other message signal. It is unlikely to be invasive, since mobile phone users set the volume of their phone according to their preference. An ongoing informal study in Ireland provided an automatic texting service for adolescents which sent automatic sms replies to adolescents' phones. Some adolescents used the service to receive a new message signal to indicate to peers that they were popular (pers. comm.).

Mobile ownership figures indicate that using a mobile phone in public is a common and 
accepted activity. Filling in a mobile diary could be indistinguishable to an observer from composing a text message or playing a game. A mobile diary might be completed unobtrusively by adolescent clients offering them sufficient privacy to chart their moods. The mood information recorded by a client can be easily and immediately sent to secure storage, preventing the loss of information. The client's phone can be password protected to prevent anyone accessing sensitive information. The privacy and security provided by the mobile phone may contribute to greater candour. Research has reported that SMS users are more likely to be intimate and revealing in text messages than in face-to-face conversations (Reid \& Reid, 2003).

Security of information and identity is of particular concern in adolescent mental health care. Software on a mobile phone can encrypt and protect client information. For example, using javame, a mobile programming language for phones, it is straightforward to ensure sufficient security by providing a password and encrypting information. There is no need to transmit sensitive client information like mobile phone numbers. It is particularly important that mood information is secure from peers and family members. Adolescents are already protective of their phones. For example, Vincent has reported in a study that adolescents kept their phones close to them to stop parents from reading text messages or siblings from stealing them (Vincent, 2004). Many adolescents feel uncomfortable when others look through their phone (International Telecommunications Union, 2004). A 2006 UK study found that $68 \%$ of $15-17$ year olds would not let their parents look through text messages or pictures on their phones (YouGov, 2006).

Diary studies typically require long training sessions (Reis \& Gable, 2000, 190-222). It is of course important to provide clear instruction on the protocols for recording mood information on any medium. It may not be necessary to provide detailed training sessions when instructing adolescents how to use their own phones. Adolescents may find entering text on their phones significantly easier and more comfortable than entering information desktop computer, a PDA or even a paper chart. This could also be very important for clients with literacy difficulties.

There are significant issues related to the introduction of mobile phones in therapy that have to be taken into consideration. The cost of data transmission is an issue that therapeutic services would have to address should mobile charting (or any mobile phone-based activity) be introduced on a day-to-day basis. There are several options for the transmission of information from a mobile phone. The most convenient method for, the client and the practitioner, is to send charted information over the mobile network. On average, this would amount to the cost of one or two text messages a week. There are other solutions available which involve no cost. It is possible to use bluetooth or infrared in a therapy session to send charted information to a desktop computer. However, these cost-free solutions are a less convenient solution which would entail practitioners getting involved in technical matters.

The mobile phone has more severe limitations on input and display than other mobile devices like PDAs. This restricts the range of content and types of activities that are suitable for mobile phones. When designing charts for adolescents it is important to keep charts short and appealing. In order to maximize participant commitment it has been recommended to keep diaries short and to require less than a few minutes to complete (Bolger et al., 2003, 579-616). Palmblad and Tiplady have made several recommendations for the design of mobile user interfaces for electronic patient reported outcomes (ePRO) which are informative (Palmblad \& Tiplady, 2004). While their suggestions are directed toward general patient use, particularly on PDAs, many are relevant here. For example, they argue that questions presented to the user should require an active response and that all attempts should be made to keep content on one mobile screen to require no scrolling. 
In summary, the mobile phone may provide adolescents with greater convenience, privacy and security than paper diaries which may result in overall greater compliance. The mobile phone is a constant companion for a high percentage of adolescents and is therefore accessible almost everywhere. It is a low cost and client-centred platform which would seem suited to therapeutic services. The following section presents a pilot study comparing the use of a paper mood diary with a newly developed mobile phone mood diary.

\section{Mobile Mood Chart Pilot Study}

\section{Method}

The main purpose of this study is to examine the use of a mobile phone as a platform for engaging adolescents in therapeutic activities, particularly mood charting. This pilot study investigated whether mobile phones are more effective than pen and paper methods for mood charting. Efficacy was primarily judged on compliance as well as other contributing factors like security, privacy, ease of use and convenience. Information was also gathered on adolescent attitudes to mobile phones being used for this purpose. All procedures in this study were approved by the Mater Hospital ethics board.

\section{Paper \& Mobile Diary Design}

The authors designed the paper and mobile chart used in this study. Both were designed in consultation with practitioners to get feedback on their suitability. Significant effort was made to make both diaries similar other self-reporting mood charts for adolescents. Paper and mobile diaries both allowed participants to record the following: date, energy, mood, sleep and textual thoughts or feelings (see figure $1 \& 2$ ). The two methods were deliberately made very similar in terms of graphics and use of colour. Both charts use a 1-10 numerical scale for rating mood and energy. Sleep is recorded in hours and there is a free area to enter any thoughts.

\begin{tabular}{|c|c|c|c|c|}
\hline $\begin{array}{l}\text { Date } \\
\text { \&Time }\end{array}$ & $\begin{array}{c}\text { Energy } \\
\text { How much energy do } \\
\text { you have out of ten? } \\
\text { (please put an ' } X \text { ' on } \\
\text { the scale) }\end{array}$ & $\begin{array}{c}\text { Sleep } \\
\text { How many hours } \\
\text { did you sleep last } \\
\text { night? }\end{array}$ & $\begin{array}{c}\text { Mood } \\
\text { How do you feel today } \\
\text { out of ten? } \\
\text { (please put an 'X' on the } \\
\text { scale) }\end{array}$ & $\begin{array}{c}\text { Diary - please describe how } \\
\text { you feel today. }\end{array}$ \\
\hline \multirow[t]{2}{*}{$\begin{array}{r}1 / 05 / 06 \\
10.15\end{array}$} & $\begin{array}{rr}6122345678) & 0 \\
\text { very } & \text { very } \\
\text { low } & \text { high }\end{array}$ & I slept _ 8 _hours. & $\begin{array}{c}\Leftrightarrow 123456789300 \\
\text { very } \\
\text { low }\end{array}$ & $\begin{array}{l}\text { I feel great today, loads of } \\
\text { energy. }\end{array}$ \\
\hline & $\begin{array}{r}\text { 60) } 12345678910 \bigcirc \\
\text { very } \\
\text { low }\end{array}$ & I slept ___ hours. & $\begin{array}{l}\odot 12345678910 \bigcirc \\
\text { very } \\
\text { low }\end{array}$ & \\
\hline
\end{tabular}

Figure 1 A screenshot from the paper Mood Diary.

The mobile mood diary can be installed on the vast majority of mobile phones currently in circulation. It functions and behaves like other programs (e.g. phonebook, photo gallery) on a client's mobile phone. It adapts to whichever phone it is installed on, acting in a way which the client is already familiar. For example, entering text using the mobile diary is achieved in the same way as composing a text message on their phone. 

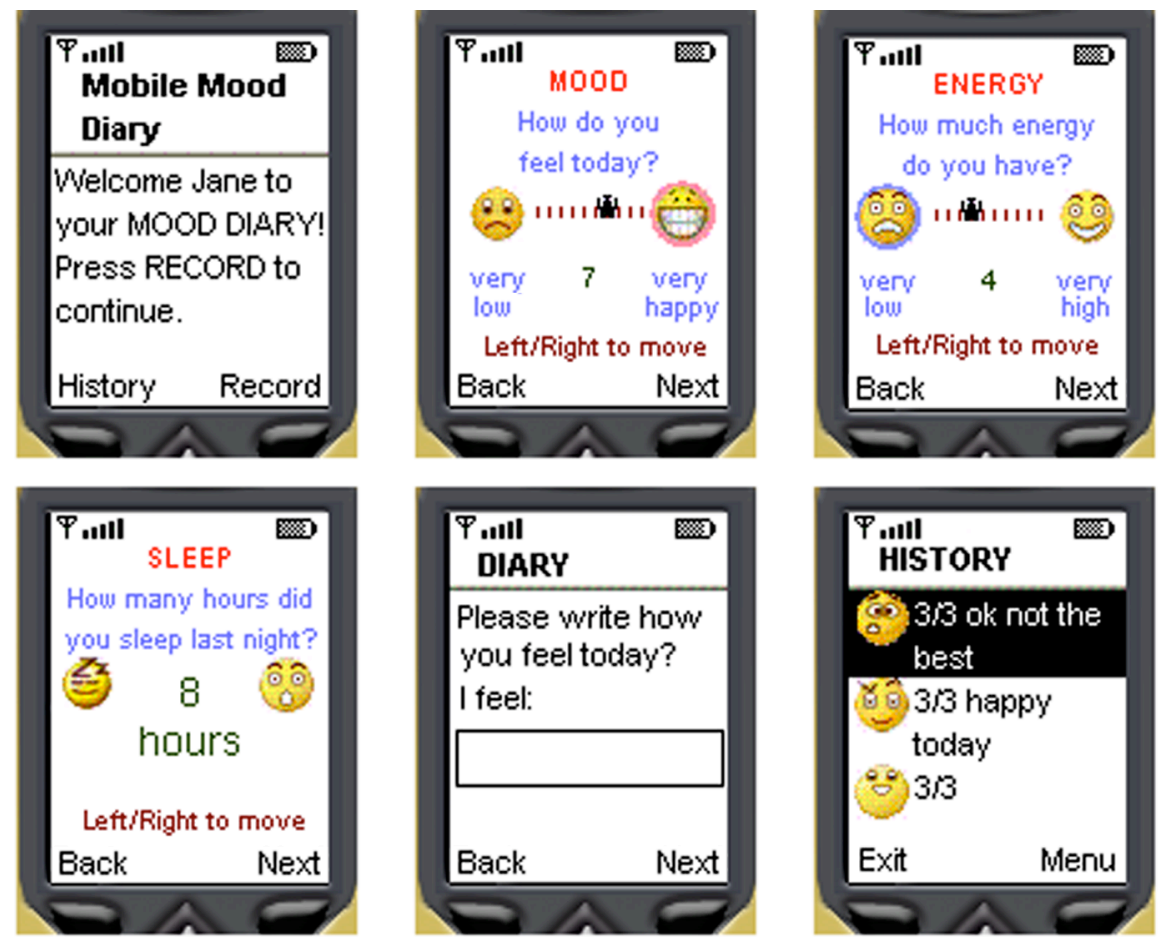

Figure 2 Six screens from the mobile Mood Diary.

This study did not use a reminder system for mobile diaries as the impact of such a feature requires a separate study. It was decided not to implement a password on the mobile diary. Since there was no means of contacting researchers during the study, it was deemed necessary to omit this feature in case a participant forgot their password and was locked out of their mobile diary for the duration of the study. In a clinical situation it would be straightforward to set up a system which would send a forgotten the password to the client or could send an automated reminder message at a regular time.

\section{Participants \& Measures}

This study took place in three schools in Dublin, Ireland between April-May, 2006. A nonclinical sample of 73 self-selecting students took part in the study with school and parental permission. Participants' ages ranged from 13 to $17(n=73$ mean $=14.87 S D=1.141)$ and were mostly female (86.3\%). 52 students (71\%) were given paper-based charts and 21 students $(29 \%)$ had the mood diary loaded onto their phones. None of the participants were suffering from apparent mental health problems but all were from similar socio-economic backgrounds to adolescents attending the Mater child and adolescent clinic. One school was randomly selected for the paper study and the other two schools had classes randomly assigned to paper or mobile. To avoid giving a strong financial incentive (i.e. mobile phone credit) to mobile phone participants, the mobile diary was only installed on phones where a simple and cost-free solution was available for retrieving data. This was dependent on the brand and model of the phone and was unrelated to the cost of the phone. As a result the number of mobile participants was lower than paper participants. Installation involved loading the software onto the phone in a face-toface situation using either bluetooth, infrared or a connection cable. Brief instructions were given to each group explaining how to record an entry. Participants were instructed to record one mood at any time each day. This was used as the measure of compliance. They were told that if 
they forgot to record an entry on one particular day then to move on to the next day and not record a mood for the missed day.

Testing lasted for two weeks. No incentive was given to either group and no training sessions were given. There was no contact between researchers and participants during the two week testing period, and no participants were reminded to use their charts. There was no contact with the mobile group and no technical support was provided to either group. All students were informed that if they felt upset during testing to contact the school counsellor.

At the end of testing all data was collected in person. Participants were given an eleven element questionnaire covering their use of the mood chart, their impressions and their ideas for improvements. 70 questionnaires (96\%) were collected from participants. Only 18 paper diaries were recovered (35\% of paper group). The rest were either forgotten at home or lost. Mood charts from 17 mobile diary participants were collected ( $81 \%$ of mobile group). Adolescents who had opted not to participate in the full study completed a general survey about their use of mobile phones and their attitudes toward them.

\section{$\underline{\text { Results }}$}

\section{Compliance}

In order to assess whether a paper versus mobile phone based platform influenced compliance behaviours, participants who had completed paper based diaries $(n=16)$ and participants who had completed a mobile phone based diary $(n=17)$ were assigned to two groups. Compliance was judged as completing at least one entry per day. Actual compliance for the paper diaries was based on filtering the most obvious cases of hoarding where participants directly admitted filling in all entries retrospectively. The mobile diary group automatically had their entries time stamped and therefore actual compliance was transparent. Data was analysed using an independent samples t-test in order to test for significant differences in the number of mood entries participants completed. Groups were found to differ significantly in their rates of actual compliance $t=-2.324, p<.027$, with the mobile phone based diary group (mean=8.12) producing significantly more entries than the paper based diary group (mean=5.44).

Due to the significant amount of lost or forgotten diaries and the difficulty in identifying cases of hoarding it is difficult to get an accurate overall picture of compliance in the paperbased diary group. Previous research would indicate that actual compliance would be considerably lower than reported compliance. For example in a study which appraised paper compliance using a specially developed paper diary, adult participants' reported compliance was $90 \%$ while actual compliance was as low as $11 \%$. This study also found that most participants engaged in hoarding (Stone et al., 2002, 1193-1194). Based on teachers' comments, questionnaires from paper diary participants and interviews, we believe that this occurred in this study also. It became clear that many participants copied entries and dates from each other as well as filling out a lot of entries just before collection. For example several participants admitted (in discussion and in questionnaires) parking-lot compliance, filling out most of their entries just before data was collected. Comments like 'I filled loads in one go', 'I did them all today' were common. In one class $(n=12)$ a teacher mentioned that participants from the paperbased group were "copying from each other" before the diaries were collected.

The statistically significant difference in compliance for the mobile diary group supports the hypothesis that the mobile phone is a suitable platform for mood charting. 
Privacy and Security

In order to test whether there was a significant difference in participants' perceived privacy between phone and paper based groups while completing a mood entry, participants in the paper group $(n=48)$ were compared to participants in the phone group $(n=19)$. Phone participants reported feeling more privacy when recording a mood than paper participants. 18 phone participants (95\%) felt they had sufficient privacy and 1 did not (5\%). Out of the paper group, 41 $(85 \%)$ felt they had enough privacy and $7(15 \%)$ did not. Comments made by both groups are particularly informative on this matter. 4 comments from paper participants identified a concern about the possibility of other people observing them entering moods and possibly even what they were entering. For example, one participant said that her friend "kept stealing it". Other comments mention other people's curiosity: "people would ask 'What's that page you're filling out?", or another "I felt that other people could be reading it". Only one person in the mobile phone group commented at all on privacy, mentioning that his brother would ask him what he was doing. This is important as there was no expressed concern in the mobile group about the possibility of someone seeing what they were entering. In this regard the small personal screen of the mobile phone becomes an advantage. It is interesting to see some of the comments from users of the paper diary, expressed in the questionnaire, about why they would prefer a mobile diary if given the choice. Several people thought that the phone would be more private: "You can conceal more easily so there is more privacy" or that "it would be much more private".

No significant difference was found between the perceived security of recorded moods for paper and phone-based diaries. This finding was expected since no password facility was provided for the mobile diary. Mobile participants expressed concern that "if someone went into games they might think it was a game and find out about how you felt". Four of the five mobile participants who commented on this felt that their entries were not sufficiently secure and that there should be a password - "It would have been better if I could set a lock code on it". In a study with a password protected mobile diary there may be a significant improvement in participants' response to this question. It is a simple matter to implement a password on the mobile phone, while it is not possible to do so for a paper diary. The password would be something memorable to the client, such as their mobile phone pin code, which if lost is easily recovered.

The fact that so few paper diaries were recovered is indicative that paper diaries are liable to be misplaced or lost. 4 comments from the mobile group reflected the idea that 'you can't lose it (the phone)' and that 'it would be safer'.

This study revealed trust issues relating to how the software was described to adolescents. At the outset, experimenters told participants they would be 'installing some software' on their mobile phones. This particular phrase made some adolescents suspicious of the testing, and potentially afraid they were being screened for mental health problems or that the software might "damage" their phones. This latter issue is most likely as a result of using participants' own mobile phones. Describing the installation as a "download" like a game or a ringtone rather than a program or an application was sufficient to allay such fears. The context and type of language used to describe new technologies to adolescents is important - protocols which deal with these language and context issues would help experimenters and practitioners when they are introducing new technologies to adolescents. 
Remembering and Availability

The mobile phone participants (mean $=2.58 s d=1.121$ where $1=$ very easy $5=$ very difficult) felt that it was easier to remember to chart than the paper participants (mean=3.15 $s d=1.185)$ in this study, but this result was only approaching significance ( $p>.078$ under independent samples t-test).

It is interesting that an unmotivated non-clinical group of adolescents should find it easier to remember to record their moods using a mobile phone than paper. This is corroborated by the actual compliance rates discussed above. It is not immediately evident why mobile participants found it easier to remember to chart. It is may be related to the availability of the device. Adolescents frequently have their phones with them, while they rarely carry pen and paper around with them. $68.4 \%$ of adolescents from the general survey group $(\mathrm{n}=20)$ said they had their phone with them "always", while a further $20 \%$ said they carried it with them "very often". The other $10 \%$ said they carried it "rarely". If a participant remembered to record a mood and had their phone with them, then they could do so immediately. If a paper participant remembered to chart and did not have a pen and paper with them, then further effort was required to remember to chart at a later time. The following comment illustrates this point: "the phone might help me record it more frequently because I always have it with me so I wouldn't forget to fill it in."

There may also be the factor that adolescents are likely to use their phone when bored or to "play" with their phone. Participants from the paper diary group who did remember to chart and had good compliance typically were independent enough to devise their own strategy to help them to remember. For example three participants put the mood diary in their homework journal so they would remember to do it when they opened their journal.

Four participants from the mobile group $(\mathrm{n}=20)$ suggested that a reminder function would have helped them remember. One participant from the same group actually set their own alarm to help. This shows a similar independence and initiative to the paper participants who devised their own signalling system.

Ease of use

Both types of chart were considered to be easy to use with no significant difference between the two groups $(\mathrm{n}=19$, mean $=1.63$, $\mathrm{sd}=.761$ for the mobile group, and $\mathrm{n}=49$, mean $=2.06$ and $\mathrm{sd}=1.069$ for the paper group, where $1=$ very easy and $5=$ very difficult). This supports the view that the mobile diary is deemed no harder to use than the paper diary, even though the paper method is viewed as extremely straightforward. This is encouraging since no technical training sessions or technical support was provided. To our knowledge, there were no cases where adolescents did not know how to use the mobile phone chart.

For time taken to complete one mood entry the mobile phone diary group $(\mathrm{n}=19)$ perceived themselves to be quicker at completing mean $=1.26 s d=.452$ than the paper group $(\mathrm{n}=45)$ mean $=$ $1.56 s d=.624$ where $1=$ "a few moments" and $3=$ "more than 5 minutes". Again there was no significant difference, in spite of the speed with which the paper diary can be used. This demonstrates that recording a mood entry on the phone was not an involved and difficult process, and gives weight to the findings above that the mobile mood diary was straightforward to use. 


\section{Method bias}

Administration methods can affect the measures of mood (Tseng et al., 1997, 305-316). When evaluating any method for recording moods, it is therefore necessary to consider if the data collection method introduces bias into the collected data. In order to investigate if the mobile method created a bias on self-charted mood ratings all valid mood entries were compared across the two groups. The mean and standard deviation of self-charted mood ratings (on a scale of 110) between the two groups are very similar (paper: mean $=7.28 \mathrm{sd}=1.941$, phone: mean $=7.21$ $\mathrm{sd}=1.894$ ). A comparison of histograms also shows a similar distribution of mood values across the two groups.

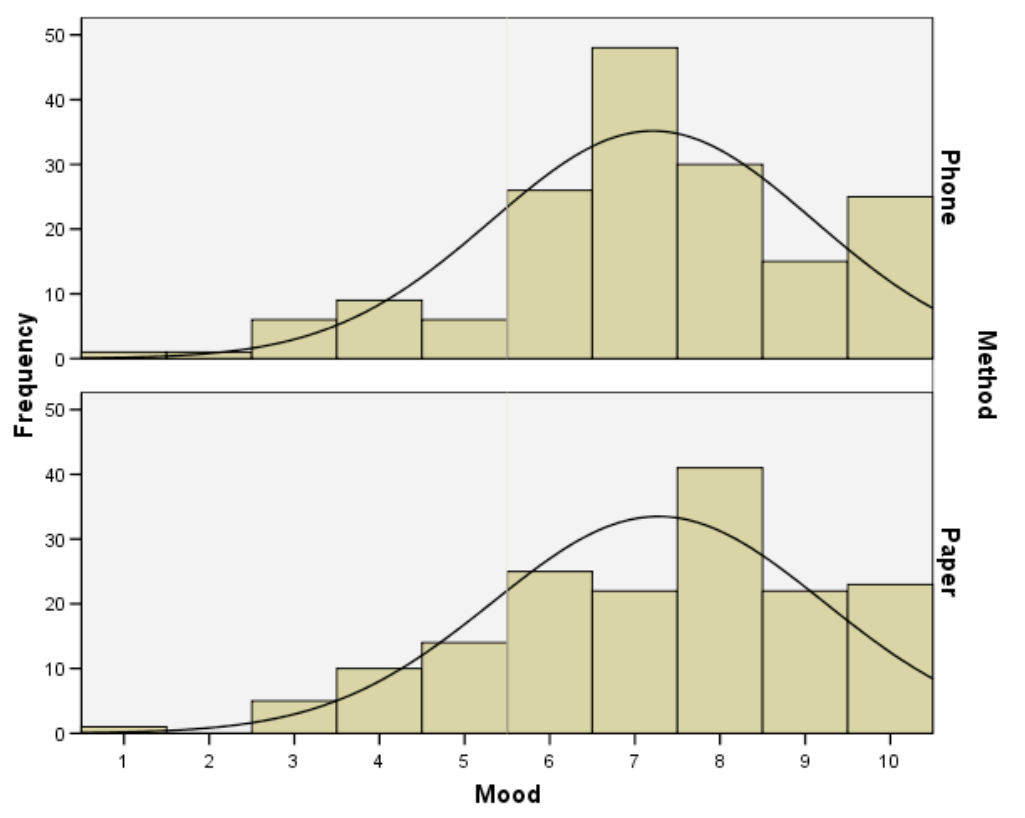

Figure 3 Histograms comparing mood ratings between groups.

Previous studies have shown that computer anxiety can affect mood ratings on desktop computers (Tseng et al., 1998, 599-610). More recently, in a study with patients with bipolar disorder, it was found that, although computer access and ability are associated with bias, that using a computer did not appear to bias the collected data (M. Bauer et al., 2005, 1-7). According to Bauer et al., this change was due to the increasing prevalence of computers. There may be fewer such issues using mobile phones as an administrative method because of their pervasiveness. Tseng et al. have recommended easy-to-use interfaces as a way of reducing problems related to method bias (Tseng et al., 1998, 599-610). Participants in this study rated the mobile mood diary as equal to a paper diary in ease-of-use. Establishing a method's ease of use does not necessarily indicate that there is no bias, but it has been a significant factor in previous studies.

\section{Preferred Method: Paper or Phone?}

There was strong agreement between groups (paper $n=43$ mobile $n=19$ ) on a preferred method for recording moods. If given a choice between a phone or paper method, $88.7 \%$ of participants would prefer to complete such tasks on their mobile phones, with $11.3 \%$ preferring paper. Similar results were obtained from the general survey group $(n=20)$ where $85 \%$ would 
prefer their mobile phones and $15 \%$ paper methods. This supports the proposition that many adolescents find the mobile phone to be a more appealing platform for this type of activity. Some comments illustrate this: "things on paper seem like it's an assignment - more likely not to do it. Having it on the mobile made it less formal and it didn't seem like work".

\section{Other findings}

As discussed above, paper participants hoarded mood entries. Analysis of the mobile phone entries shows that there are no obvious attempts to back date entries through hoarding. Another interesting finding was that 6 mobile participants ( $\mathrm{n}=17,35 \%$ of mobile participants) had at least one day when they voluntarily recorded more than one valid mood entry. This is particularly interesting because participants were only instructed to complete one mood entry a day. Out of a total 172 mobile phone entries, $32(19 \%)$ were extra entries not registered under compliance. Days with more than one entry are spread over the testing period which would indicate that it is not related to the adolescent learning how to use the program or to a short-lived novelty factor. Limitations and Future Work

This was an exploratory study, which identified many interesting points relating to the mobile phone as a therapeutic platform. Many of the findings are necessarily tentative due to the sample size and gender bias of the study. Future research is needed in the areas identified above such as mobile privacy, security and in general into the mobile phone as a therapeutic platform. A study involving a larger male sample, with a greater sample size, should also be undertaken. The findings from this study are limited by the fact that participants were not suffering from mental health problems. A clinical evaluation of the mobile mood chart with adolescent participants would help provide more pertinent data regarding its use in clinical situations. In this initial study, the mood ratings of the two participating groups did not appear to be biased. A more detailed study, including independent assessments from clinical practitioners and other measures, is necessary to investigate bias associated with the mobile method. It would also be interesting to undertake a comparative study of mobile phones and PDAs for recording moods. Once these studies have been completed it would be useful to examine how far these findings are similar across different age groups and different socio-economic backgrounds. Finally, considering the limits of mobile textual input, it would be very interesting to explore the therapeutic value of different types of input, like photographs or audio. Different inputs may have interesting and varied therapeutic use and help to overcome the mobile phone's present limitations.

The long term objective of this research is to explore the potential value of the mobile phone as a tool which therapeutic services may use as a means of engaging clients and as a way to enable clients to more easily record personal content. Clinical trials with adolescents attending therapeutic sessions are currently under way. The mood diary for these trials includes a password and reminder system for the participating adolescents. The mood information charted is available through an Internet browser which allows the practitioners and client to view graphs of mood entries in a session. It also allows the practitioner and client to compose a reminder text message together which is sent to the mobile phone each day at a chosen time.

\section{Conclusion}

This paper has presented evidence that the mobile phone may be an effective platform for allowing many adolescents to record their moods, although some may still prefer other methods like paper charts. The study found that adolescents are more likely to complete a mood diary that is presented on a mobile phone than when a similar diary is presented as a paper chart. The 
mobile phone diary was able to appraise compliance. It provided adolescents with sufficient privacy, was cost effective, user-friendly and client-centred. This paper argued that a personal mobile device, like the mobile phone has particular advantages for many adolescents and clinics over other mobile devices. The mobile phone has its limitations, like the cost of data transmission and screen size which need to be taken into consideration. Furthermore, the mobile phone is not for all situations or for all clients. It should be seen as another tool available to practitioners for use where suitable. Although this study was limited by the sample size and gender bias, it revealed a number of interesting issues which deserve further study.

\section{Acknowledgements}

This research has been funded by the Higher Education Authority of Ireland. The authors would like to acknowledge the generous help and input of David Coyle, Paul O' Donoghue, David Martin, Carol Downey, Sister Theresa, Bride Coyle, St. Mary's Secondary School, Mount Temple Comprehensive School and Mount Carmel Secondary School.

\section{References}

ASSAY, T. P., \& LAMBERT, M. J. (1999). The Empirical Case for Common Factors in Therapy: Quantitative Findings. In B. L. Duncan, M. L. Hubble \& S. D. Miller (Eds.), The Heart and Soul of Change (pp. 23-55). Washington, DC: American Psychological Association.

BAUER, M., GROF, P., GYULAI, L., RASGON, N., GLENN, T., \& WHYBROW, P. C. (2004). Using technology to improve longitudinal studies: self-reporting with ChronoRecord in bipolar disorder. Bipolar Disorders, 6(1), 6774.

BAUER, M., RASGON, N., GROF, P., GYULAI, L., \& GLENN, T. (2005). Does the Use of an Automated Tool for Self-Reporting Mood by Patients With Bipolar Disorder Bias the Collected Data? Medscape General Medicine, $7(21), 1-7$.

BAUER, S., PERCEVIC, R., OKON, E., MEERMANN, R., \& KORDY, H. (2003). Use of Text Messaging in the Aftercare of Patients with Bulimia Nervosa. European Eating Disorders Review, 11, 279-290.

BOLGER, N., DAVIS, A., \& RAFAELI, E. (2003). Diary Methods: Capturing Life as it is Lived. Annual Review of Psychology, 54(1), 579-616.

CRABTREE, J., NATHAN, M., \& ROBERTS, S. (2003). MobileUK: Mobile Phones and Everyday LIfe. London: The Work Foundation.

FELDMAN BARRETT, L., \& BARRETT, D. J. (2001). An introduction to computerized experience sampling in psychology. Soc. Sci. Comput. Rev., 19, 175-185.

GESER, H. (2006). Pre-teen cell phone adoption: consequences for later patterns of phone usage and involvement. Zürich.

HENKER, B., WHALEN, C. K., JAMNER, L. D., \& DELFINO, R. J. (2002). Anxiety, Affect and Activity in Teenagers: Monitoring Daily Life With Electronic Diaries. Child and Adolescent Psychiatry, 41(6), 660-670.

INTERNATIONAL TELECOMMUNICATIONS UNION. (2004). Social and Human Considerations for a more Mobile World Background Paper.

KOOP, A. (2002). Lessons learned from 16 years usage of mobile computers in clinical trials. Mobile Computing in Medicine, 15, 9-24.

LEVERICH, G. S., \& POST, R. M. (1996). Life charting the course of bipolar disorder. Current Review of Mood and Anxiety Disorders, 1, 48-61.

LING, R. (2001). Adolescent girls and young adult men: Two sub-cultures of the mobile telephone. Kjeller: Telenor Research and development. 
NORTON, M., WONDERLICH, S. A., MYERS, T., MITCHELL, J. E., \& CROSBY, R. D. (2003). The Use of Palmtop Computers in the Treatment of Bulimia Nervosa. European Eating Disorders Review, 11, 231-242.

OFFER, D., HOWARD, K. I., SCHONERT, K. A. AND OSTROV, E. (1991). "To whom do adolescents turn for help? Differences between disturbed and non-disturbed adolescents." Journal of the American Academy of Child and Adolescent Psychiatry, 30(4) pp. 623-630.

POST, R. M., NOLEN, W. A., KUPKA, R. W., DENICOFF, K. D., LEVERICH, G. S., KECK, P. E., JR., et al. (2001). The Stanley Foundation Bipolar Network: 1. Rationale and methods. British Journal of Psychiatry, 178(41), 169-176.

RAYMOND, S. A., \& ROSS, R. N. (2000). Electronic Subject Diaries in Clinical Trials. Applied Clinical Trials, 48-57.

REID, D., \& REID, F. J. M. (2003). Text mates and text circles: insights into the social ecology of SMS text messaging. Paper presented at the The Mobile Revolution: A Retrospective-Lesson on Social Shaping, Proceedings of the 4th Wireless World Conference.

REIS, H. T., \& GABLE, S. T. (2000). Event-sampling and other methods for studying everyday experience. In H. T. Reis \& M. C. Judd (Eds.), Handbook of Research Methods in Social and Personality Psychology (pp. 190-222). New York: Cambridge University Press.

SHIFFMAN, S., HUFFORD, M., HICKCOX, M., PATY, J. A., M., G., \& KASSEL, J. D. (1997). Remember that? A comparison of real time versus retrospective recall of smoking lapses. Journal of Consulting and Clinical Psychology, 65, 292-300.

STONE, A. A., SHIfFMAN, S., SCHWARTZ, J. E., BRODERICK, J. E., \& HUFFORD, M. R. (2002). Patient non-compliance with paper diaries. BMJ, 324(1), 1193-1194.

TSENG, H.-M., TIPLADY, B., MACLEOD, H. A., \& WRIGHT, P. (1997). Computer anxiety and measurement of mood change. Computers in Human Behavior, 13, 305-316.

TSENG, H.-M., TIPLADY, B., MACLEOD, H. A., \& WRIGHT, P. (1998). Computer anxiety: A comparison of pen-based personal digital assistants, conventional computer and paper assessments of mood and performance. British Journal of Psychology, 89, 599-610.

US SURGEON GENERAL. (1999). Mental Health: A Report of the Surgeon General. Rockville, MD: US Department of Health and Human Services.

VAN VEEN, N. (2007). How European Youth Use Mobile: Catering To The Whims Of 50 Million Mobile Users: Forrester Research.

VANDENKERKHOF, E. G., GOLDSTEIN, D. H., BLAINE, W. C., \& RIMMER, M. J. (2005). A Comparison of Paper with Electronic Patient-Completed Questionnaires in a Preoperative Clinic. Anesthesia and Analgesia, 101(4), 1075-1080.

VINCENT, J. (2004). '11 16 Mobile’ Examining mobile phone and ICT use amongst children aged 11 to 16. Guildford: University of Surrey.

YOUGOV. (2006). The Mobile Life Youth Report. Retrieved 05/03/2007, from http://www.yougov.com/archives/pdf/CPW060101004_2.pdf 